\title{
Avaliação das condições higiênico-sanitárias em embarcações mistas da região Amazônica
}

\section{Evaluation of the hygienic and sanitary conditions in mixed vessels of the Amazon region}

\author{
Ariane Kluczkovskil,* iD
}

Samir Pinto'

Augusto Kluczkovski-Junior"

Marco Pinto"

Jerfeson Caldas"II

Andrea Waichman' iD
1 Universidade Federal do Amazonas (UFAM), Manaus, AM, Brasil

" Fundação de Vigilância em Saúde do Amazonas, Manaus, AM, Brasil

II' Agência Nacional de Vigilância Sanitária (Anvisa), Brasília, DF, Brasil

\section{RESUMO}

Introdução: A condição higiênico-sanitária de embarcações que transportam passageiros e cargas requer diagnóstico e monitoramento constantes para prevenção de doenças. Objetivo: Identificar perigos sanitários nas condições de preparação de alimentos e potabilidade de água em embarcações mistas em Manaus, Amazonas. Método: Foram analisadas 33 embarcações com análise de detecção qualitativa de Escherichia coli por substrato cromogênico para potabilidade da água e avaliação ambiental com aplicação de questionário. Resultados: Havia contaminação microbiológica em 13 (39,39\%) amostras de água de bebedouro e $31(93,93 \%)$ amostras de pia para $E$. coli. As condições estruturais e operacionais das embarcações em maioria não atendiam aos percentuais mínimos de segurança na produção de alimentos. Conclusões: Esse é o primeiro relato sobre as condições estruturais e operacionais da preparação de alimentos em embarcações mistas em Manaus e conclui-se que é necessária intervenção com políticas públicas quanto às questões higiênico-sanitárias que envolvam o fornecimento das águas servidas nas embarcações para proteção da saúde de passageiros, capacitação dos manipuladores de alimentos e inspeção de ambientes.

PALAVRAS-CHAVE: Coliforme; Potabilidade; Segurança de Alimentos

\footnotetext{
* E-mail: mendonca-ariane@hotmail.com
} 


\section{INTRODUÇ̃̃O}

O gerenciamento de risco de alimentos é uma ferramenta fundamental em muitos países e requer a identificação dos perigos existentes sejam de origem ambiental ou operacional ${ }^{1,2}$. Na região Amazônica, o transporte fluvial é de relevância para a população, já que os rios são considerados estradas na ausência de meio de transporte terrestre. Portanto, as condições higiênico-sanitárias das cozinhas e a potabilidade de água nas embarcações mistas, que transportam passageiros e cargas, são aspectos que devem ser monitorados para prevenir doenças.

Segundo a World Health Organization, a avaliação de risco alimentar pode ser definida como uma avaliação científica de efeitos adversos à saúde conhecidos ou potenciais, resultantes da exposição humana a perigos de origem alimentar. Esse processo se constitui da identificação do perigo, caracterização do perigo, exposição ao risco e caracterização do risco ${ }^{3}$. Dentre os perigos biológicos estão bactérias patogênicas que podem contaminar os alimentos e a água causando as doenças transmitidas por alimentos (DTA). Existem mais de 250 tipos de DTA no mundo, sendo que a maioria delas são infecções causadas por bactérias e suas toxinas; vírus e outros parasitas ${ }^{4}$. Diversos microrganismos podem contaminar os alimentos, portanto há diferentes formas de contaminação $0^{5}$. Nesse contexto, as condições higiênico-sanitárias das áreas de manipulação e armazenamento de alimentos, bem como a potabilidade da água de consumo, podem ser formas de transmissão se não atenderem aos limites sanitários que visam proteger a saúde.

Os surtos alimentares resultam da associação entre o consumo de alimentos contaminados por meio da manipulação inadequada e conservação ou distribuição em condições impróprias ${ }^{6}$. Em relação às embarcações, a legislação brasileira possui um guia para navios de cruzeiro, que possuem estrutura de maior porte e complexidade diferentes das embarcações regionais da Amazônia. Rooney et al. ${ }^{7}$ relataram que em surtos de navios havia frequência elevada de Salmonella sp. relacionada aos alimentos que, como bactéria patogênica, colocava em risco a saúde dos passageiros e tripulantes. No entanto, na região Amazônica são escassos os dados relacionados às condições sanitárias de embarcações que manipulam alimentos com transporte de passageiros e cargas. Nessa região, os relatos de DTA estão relacionados com alimentos comercializados, como, por exemplo: o queijo tipo coalho contaminado por toxina estafilocócica ${ }^{8}$ e a transmissão oral de doença de chagas pelo consumo de açaí?. Nesse contexto, as embarcações podem ser locais de surtos alimentares, com origem na falta de boas práticas de manipulação (BPM), que são regras de higiene a serem aplicadas mesmo em meio de transporte fluvial ou por passageiros vetores de enfermidades, como vírus que podem ser transmitidos por condições sanitárias ambientais inadequadas, ou por se tratar de espaços confinados como os navios $^{10}$. Um outro aspecto que pode ser um perigo e comprometer a saúde dos passageiros é a potabilidade da água de consumo das embarcações. Finger et al. ${ }^{11}$ relataram que, dentre os surtos registrados oficialmente no Brasil de 2000 a 2018, $12 \%$ estavam relacionados com contaminação da água. Em estudo em embarcações da região Amazônica, de Paula et al. ${ }^{12}$ avaliaram que a água consumida pelos passageiros e tripulantes era de péssima qualidade, apresentando $89 \%$ de amostras contaminadas por coliformes totais e $84 \%$ pela bactéria Escherichia coli. No Brasil, a água considerada potável deve estar em conformidade com padrão recomendado pela Portaria de Consolidação $n^{\circ} 5$, de 28 de setembro de $2017^{13}$, do Ministério da Saúde, que define os parâmetros físico-químicos e microbiológicos que garantem a potabilidade da água, no entanto, na região Amazônica, muitas embarcações mistas adquirem águas subterrâneas (poço) fornecidas por empresas em que as bombonas ou garrafões são preenchidos e transportados para os barcos. Diante do contexto da falta de dados relativos às condições higiênico-sanitárias em embarcações mistas e para contribuir com a análise de perigos na definição e implantação de políticas públicas de proteção à saúde, o objetivo desse trabalho foi verificar a potabilidade de água, bem como aspectos de higiene e as condições ambientais de preparação de alimentos nas embarcações mistas de portos de Manaus, Amazonas (AM).

\section{MÉTODO}

\section{Amostragem}

Foram estudadas embarcações mistas $(n=33)$ dos portos de Manaus no período de 2018 a 2019. Nas embarcações foi aplicada a inspeção visual por fiscais do órgão estadual de Vigilância Sanitária (Visa), considerando que a aplicação da inspeção é uma das ferramentas eficazes para o levantamento de informações para análise de riscos ${ }^{14,15}$. Para avaliação da higiene dos ambientes, foi aplicado um questionário com itens de roteiro de inspeção, baseados na legislação vigente para manipulação de alimentos $^{16,17}$. Por observação visual foram avaliadas as condições de: (a) equipamentos de refrigeração (câmara fria, freezer ou geladeira); (b) ambientes e (c) manipuladores de alimentos, nas cozinhas quanto às BPM.

Potabilidade: (a) Detecção qualitativa de Escherichia coli: foi utilizado o Kit Colilert ${ }^{\circledR}$ (IDDEX), substrato cromogênico. A análise foi feita dentro de capela de fluxo laminar e, em cada amostra em frasco de $100 \mathrm{ml}$, feita assepsia do frasco com algodão embebido em álcool, transferida para um frasco estéril graduado. Em seguida adicionou-se a cada frasco um flaconete contendo a quantidade exata de meio de cultura e incubou-se a $35 \pm 0,5^{\circ} \mathrm{C}$ for $24 \mathrm{~h}$. Após incubação, observou-se a amostra em luz UV 365-nm quanto à presença de fluorescência em amostras positivas; (b) $\mathrm{pH}$ : as amostras também foram avaliadas quanto ao potencial hidrogeniônico por meio de potenciômetro, conforme $\mathrm{APHA}^{18}$. Apesar de a legislação brasileira para potabilidade de água citar outros ensaios laboratoriais, foram apenas executados os ensaios citados acima, (a) e (b), devido à previsão de recursos que viabilizou a execução do projeto ter sido limitada (Chamada Conselho Nacional de Desenvolvimento Científico e Tecnológico CNPq/Agência Nacional de Vigilância Sanitária - Anvisa/Pesquisa em vigilância sanitária - Processo: 441816/2017-7). 


\section{RESULTADOS E DISCUSSÃO}

\section{Equipamentos de refrigeração}

Em 79,41\% das embarcações mistas avaliadas foi verificada a presença de freezer e somente $55,88 \%$ delas possuíam câmara fria (Figura 1). Esse dado é relevante no sentido de que gera um alerta da possível falta de temperatura adequada no transporte de alimentos tanto para consumo durante as viagens quanto para cargas que necessitem de ambiente de câmara fria. Quanto às condições de higienização dos ambientes (câmara fria, freezer e geladeira), em $90,91 \%$ havia limpeza adequada e $87,88 \%$ podiam ser higienizados, ou seja, com superfície lisa, pintura impermeável e sem madeira (Figura 2).

Apesar de todas as embarcações possuírem banheiros para passageiros e tripulantes, apenas a classificação unissex era utilizada em $100,00 \%$ das embarcações. A classificação feminino ou masculino não era utilizada por todos. Foi possível identificar que $100,00 \%$ das embarcações possuíam dispositivos de armazenamento de resíduos (por exemplo: lixeiras, bombonas e área de lixo refrigerado). Os resíduos sólidos seriam descartados somente em terra firme, na chegada ao porto de destino; e as águas servidas, segundo relatos, seriam destinadas diretamente nos rios.
A Figura 3 mostra que $81,82 \%$ das cozinhas não estavam limpas e organizadas e tinham presença de animais. Na inspeção foi relatada tanto a presença de animais de estimação como do ambiente do porto. Havia pias para lavagem de mãos $(93,94 \%)$, no entanto os materiais de teto, piso e parede não atendiam às questões sanitárias $(81,82 \%)$, sendo madeira o material mais frequente e em condições de desgaste, com acúmulo de sujeira, e metal enferrujado que impedia a higienização. Todas as embarcações atendiam ao limite de passageiros.

\section{Manipuladores de alimentos}

A embarcação mista é um ambiente restrito, em que passageiros e tripulantes permanecem por longos períodos juntos na região Amazônica pode ser dias ou semanas. Eles utilizam uma área comum para o consumo de bebidas e alimentos e servem-se em buffets ou nas próprias panelas em um balcão comum ou mesa, compartilhando a mesma fonte de alimento e mesma água, e realizando interações nos locais de consumo, inclusive em momentos fora de horário de alimentação. As áreas de manipulação, cozinhas, são ambientes geralmente de espaço restrito, o que dificulta realizar operações sem que haja cruzamento de fluxos. Mesmo nessas condições peculiares, os procedimentos operacionais padrão deveriam obedecer as

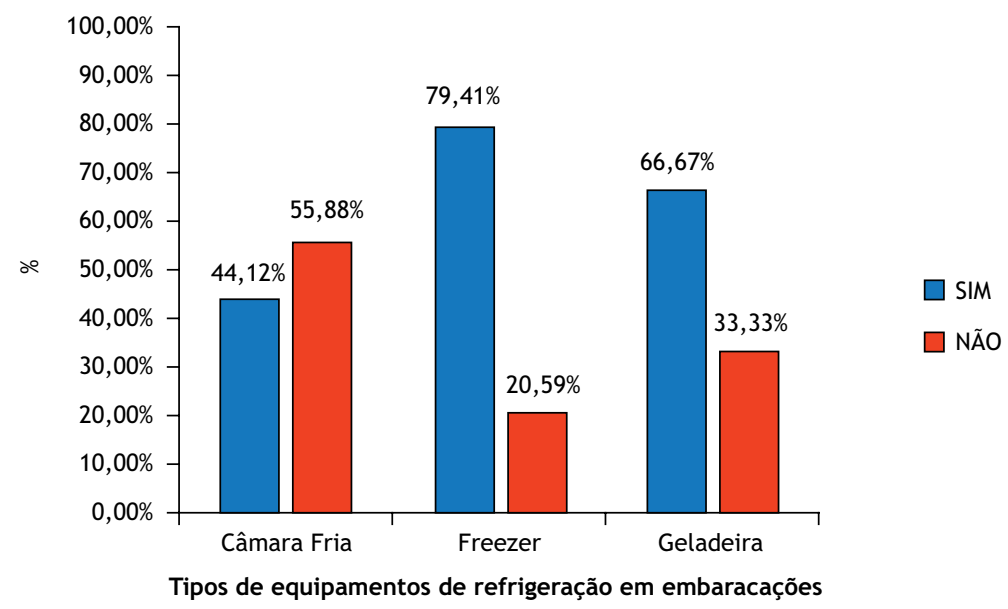

Fonte: Elaborada pelos autores, 2020.

Figura 1. Presença de equipamentos de refrigeração em embarcações mistas.

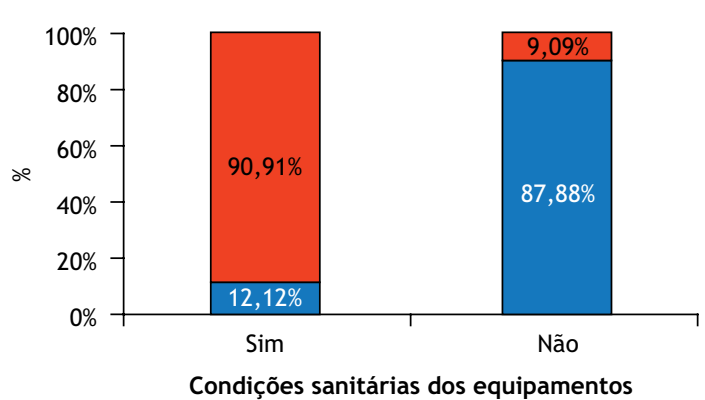

Equipamentos: Limpos e condições de uso

Equipamentos: Permitem higienização

Fonte: Elaborada pelos autores, 2020.

Figura 2. Condições dos equipamentos de refrigeração em embarcações mistas. 


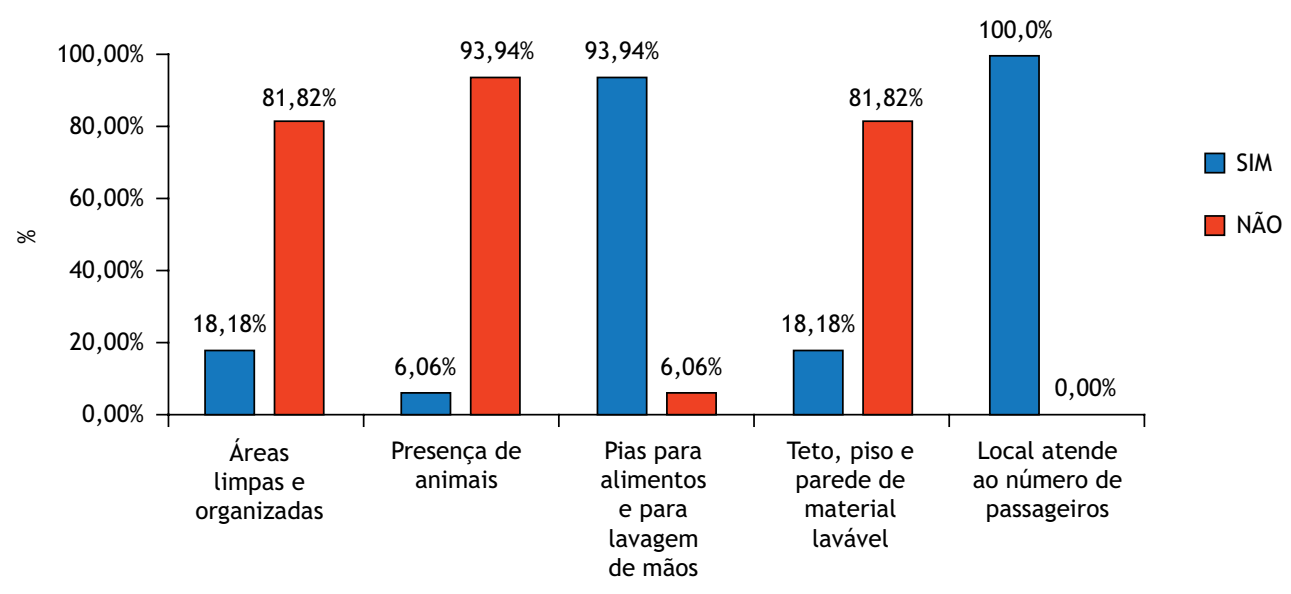

Condições dos ambientes de embarcações

Fonte: Elaborada pelos autores, 2020.

Figura 3. Condições ambientais em embarcações mistas.

BPM definidas na legislação brasileira e também o Sistema de Análise de Perigos e Pontos Críticos e Controle (APPCC) para identificar os perigos biológicos, químicos ou físicos e os pontos que afetam a produção dos alimentos de forma a mantê-los seguros ${ }^{19,20}$. Essas ferramentas de segurança na produção de alimentos já foram utilizadas com efetividade em outros países. Moutchtori et al. ${ }^{21}$ estudaram balsas que transportavam passageiros na Grécia, utilizando também questionário padronizado, com foco em identificar a utilização do APPCC na interação entre passageiros, tripulantes e alimentos e água consumida, vigilância de doenças transmissíveis, água potável, pessoal, segurança alimentar, equipamentos de limpeza, instalações sanitárias e de lavagem de mãos, gestão de substâncias perigosas e saúde ambiental. 0 resultado indicou que a maioria das embarcações avaliadas (65\%) alcançou o score de $100 \%$ de atendimento aos conceitos de APPCC. Na Itália, Capunzo et al..$^{22}$ avaliaram manipuladores de navios mercantes italianos antes e após treinamento de BPM. Os resultados mostraram melhora da qualidade da higiene dos alimentos após o curso de atualização e piora após mudança de membros da equipe de tripulação sem o treinamento. A educação contínua é necessária para garantir constantemente uma boa qualidade higiênica dos alimentos. No Brasil a legislação vigente, Resolução da Diretoria Colegiada (RDC) $n^{\circ} 72$, de 29 de dezembro de $2009^{16}$, trata das condições de saúde nos portos, no entanto vários de seus itens não são passíveis de serem executados a bordo em condições restritas, como nas embarcações mistas, e apesar de estar em vigor há mais de 10 anos é pouco conhecida entre tripulantes na região Amazônica. A RDC no 216, de 15 de setembro de $2004^{19}$, que trata de BPM em estabelecimentos, também é desconhecida entre esse grupo segundo relatos durante a aplicação do questionário de inspeção e entrevistas.

Com relação à capacitação, pode ser observado na Figura 4 que a parte operacional de execução de boas práticas quanto ao uso de equipamentos de proteção individual (EPI) era de somente $12,12 \%$ e que $87,88 \%$ das embarcações não possuíam cartazes com orientações de lavagem das mãos. Isso agrava inclusive as condições que podem causar contaminação por doenças respiratórias. No caso de prevenção de SARS-CoV-2, lavar as mãos em todas as ocasiões quando necessário, desinfetar objetos e superfícies, manter o distanciamento físico e não tocar nos olhos, nariz ou boca são recomendações básicas ${ }^{23}$. Por outro lado, o contato pessoal de longo prazo, os fluxos populacionais complexos, a falta de instalações de cuidados médicos e a infraestrutura defeituosa a bordo das embarcações podem tornar um ambiente propício para veiculação de doenças. Isso já foi observado e relatado em navios de cruzeiro por Zhang et al. ${ }^{24}$, em que os autores relataram que a lavagem das mãos é um fator importante que afeta a propagação de vírus entre os passageiros. Hadjichristodoulou et al. ${ }^{25}$ relataram que a implementação de ações corretivas após inspeções piloto aumentou o nível de conformidade com as normas de higiene em navios de passageiros navegando dentro das águas da União Europeia $\mathrm{e}$, consequentemente, melhorou as condições de higiene das embarcações. A comunicação contribuiu para o aprimoramento de identificação de surtos e melhor gestão por meio do rápido compartilhamento de informações de saúde pública, permitindo uma resposta mais oportuna e coordenada. Ao fazer-se um comparativo com os dados obtidos neste trabalho, pode-se sugerir que os meios digitais da Visa (por exemplo: homepage) divulguem textos educativos para os passageiros e tripulantes das embarcações de forma a prevenir doenças, já que a população em geral possui mais acesso à informação.

\section{Potabilidade de água}

Em todos os barcos estudados, caixas-d'água/reservatórios estavam presentes. No entanto, em nenhum $(0,00 \%)$ havia a presença de dosador de cloro. Quanto à potabilidade, foi observada a contaminação microbiológica positiva em 13 (39,39\%) amostras de água de bebedouro e $31(39,39 \%)$ foram negativas para $E$. coli (Figura 1). Segundo a legislação brasileira, E. coli deveria estar ausente em $100 \mathrm{~mL}$ de água (Figura 5). 


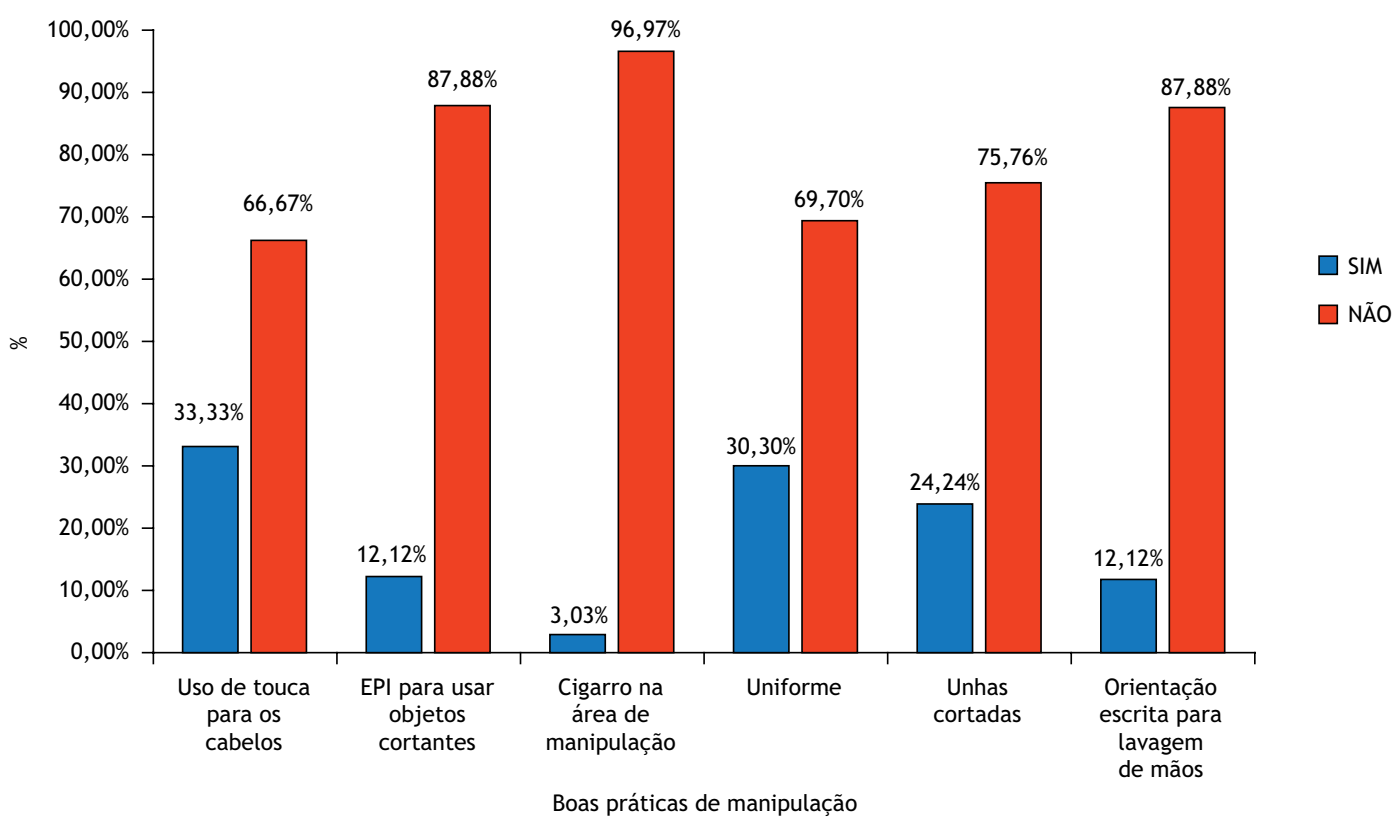

Fonte: Elaborada pelos autores, 2020.

EPI: equipamentos de proteção individual.

Figura 4. Boas práticas de manipulação entre manipuladores de alimentos em embarcações mistas.

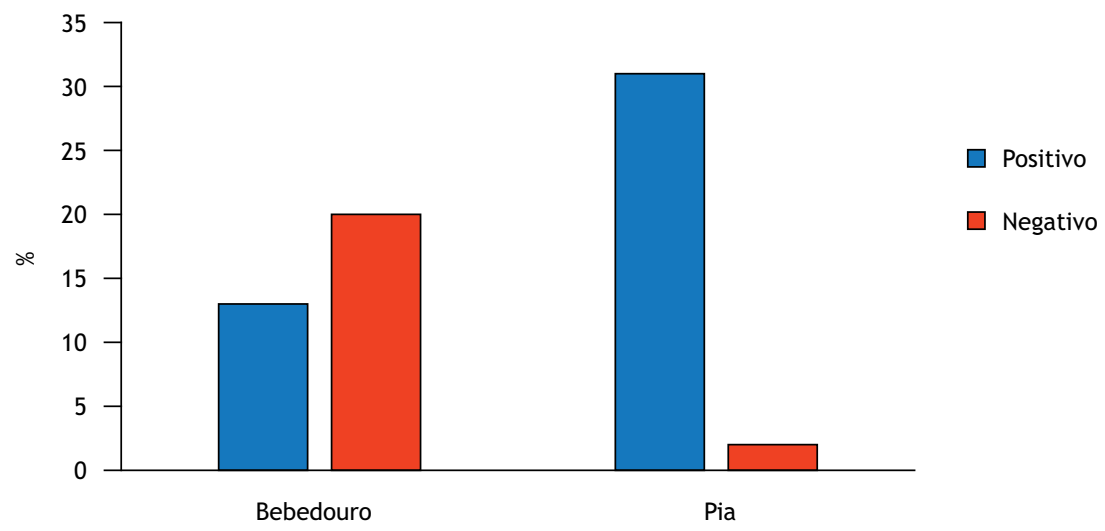

Locais de amostragem da potabilidade em embarcações

Fonte: Elaborada pelos autores, 2020.

Figura 5. Potabilidade em embarcações mistas na cidade de Manaus-AM para presença de Escherichia coli.

Aspectos sanitários em atividades portuárias na região de Manaus já haviam sido reportados por Caldas e Pozetti ${ }^{26}$ como insatisfatórios, e isso incluía a falta de potabilidade da água em $77,8 \%$ das amostras analisadas. Segundo os autores, as não conformidades encontradas poderiam estar relacionadas com a ausência de rotina de avaliação da qualidade da água, inadequação da natureza do sistema de distribuição e pontos de oferta dos portos à legislação. Neste trabalho, foi relatado pelos responsáveis pelas embarcações que o abastecimento ocorre a partir de fontes de água de poço. No estado do Amazonas, Castro et al. ${ }^{27}$ avaliaram amostras no município de Humaitá, onde não foi verificada a presença de $E$. coli em nenhuma amostra analisada. Segundo Araújo et al. ${ }^{28}$, o poço é considerado uma importante fonte de suprimento de água para consumo humano por populações que não têm acesso à rede pública de abastecimento em Manaus. As amostras do presente trabalho foram obtidas de embarcações que são abastecidas com água de poços artesianos das cidades/portos em que são atracadas e isso explicaria a contaminação das amostras de água da pia. Quanto à contaminação dos bebedouros, observamos que a utilização de reservatórios (bombonas e garrafões) com higienização deficiente pode caracterizar outra fonte de contaminação, justificando assim os resultados positivos encontrados. Portanto, seria importante a utilização de sistema de cloração da água nas embarcações de forma a garantir a potabilidade por meio de monitoramento diário do teor de cloro. Além da cloração, a correta higienização dos garrafões 
é necessária para prevenir contaminação cruzada. Infelizmente nenhuma das embarcações possuía sistema de cloração da água. A água é fundamental nas atividades de preparação de alimentos na cozinha do barco, bem como nos sistemas de higienização de utensílios e sua potabilidade deve ser mantida para evitar que seja fonte de contaminação. Por exemplo: Passos et al. ${ }^{29}$ relataram que, em amostras de água de um navio de cruzeiro, foram observados resultados positivos para coliformes a $35^{\circ} \mathrm{C}$, durante a investigação de surto por DTA.

\section{CONCLUSÕES}

Com base nas observações, conclui-se que há necessidade de definição de monitoramentos mais específicos para esse meio de transporte, por exemplo, por meio de legislação de itens de armazenamento e preparação de alimentos para fiscalização mais efetiva, bem como de avaliação mais frequente da água de consumo e do gelo. Faz-se evidente também a necessidade de política de conscientização e de sensibilização dos tripulantes quanto a conhecer e executar as BPM na preparação de alimentos. As embarcações mistas possuem peculiaridades em relação aos navios de maior porte, então é preciso uma legislação e abordagem específicas de forma a gerenciar os riscos sanitários identificados no presente trabalho. Também são necessárias formas mais eficientes de comunicação dos riscos para os passageiros e tripulantes envolvidos com o transporte em embarcações mistas na região Amazônica de forma a prevenir disseminação de doenças.

\section{REFERÊNCIAS}

1. Chartres N, Bero LA, Norris SL. A review of methods used for hazard identification and risk assessment of environmental hazards. Environ Int. 2019;123:231-9. https://doi.org/10.1016/j.envint.2018.11.060

2. Lindqvist R, Langerholc T, Ranta J, Hirvonen T, Sand S. A common approach for ranking of microbiological and chemical hazards in foods based on risk assessment-useful but is it possible? Crit Rev Food Sci Nutr. 2019:1-14. https: //doi.org/10.1080/10408398.2019.1693957

3. World Health Organization - WHO. Risk assessment. Geneva: World Health Organization; 2020[acesso 15 jun 2020]. Disponível em: https://www.who.int/foodsafety/ risk-analysis/riskassessment/en/

4. Ministério da Saúde (BR). Doenças transmitidas por alimentos: causas, sintomas, tratamento e prevenção. Brasília: Ministério da Saúde; 2019[acesso 11 nov 2020]. Disponível em: http://portalms.saude.gov.br/saude-de-a-z/ doencas-transmitidas-por-alimentos

5. Centers for Disease Control and Prevention - CDC. Foodborne germs and illnesses. Atlanta: Centers for Disease Control and Prevention; 2018[acesso 11 nov 2020]. Disponível em: https://www.cdc.gov/foodsafety/ foodborne-germs.html

6. Oliveira ABA, Paula CMD, Capalonga R, Cardoso MRI, Tondo EC. Foodborne diseases, main etiologic agents and general aspects: a review. Rev HCPA. 2010;30(3):279-85.

7. Rooney RM, Cramer EH, Mantha S, Nichols G, Bartram JK, Farber JM et al. A review of outbreaks of foodborne disease associated with passenger ships: evidence for risk management. Public Health Rep. 2004;119(4):427-34. https://doi.org/10.1016/j.phr.2004.05.007

8. Ruwer CM, Moura JF, Gonçalves MJF. Surtos de doenças transmitidas por alimentos em Manaus, Amazonas (2005-2009): o problema do queijo coalho. Segur Aliment Nutri. 2011;18(2):60-6.
9. Barbosa MDGV, Ferreira JMBB, Arcanjo ARL, Santana RAG, Magalhães LKC, Magalhães LKC et al. Chagas disease in the state of Amazonas: history, epidemiological evolution, risks of endemicity and future perspectives. Rev Soc Bras Med Trop. 2015;48(Supl.):27-33. https://doi.org/10.1590/0037-8682-0258-2013

10. Mizumoto K, Chowell G. Transmission potential of the novel coronavirus (COVID-19) onboard the diamond princess cruises ship, 2020. Infect Dis Model. 2020;5:264-70. https://doi.org/10.1016/j.idm.2020.02.003

11. Finger JA, Baroni WS, Maffei DF, Bastos DH, Pinto UM. Overview of foodborne disease outbreaks in Brazil from 2000 to 2018. Foods. 2019;8(10):434. https://doi.org/10.3390/foods8100434

12. Paula DLM, Lima ACM, Vinagre MVA, Pontes NA. Saneamento nas embarcações fluviais de passageiros na Amazônia: uma análise de risco ao meio ambiente e à saúde por meio da lógica fuzzy. Eng Sanit Ambient. 2019;24(2):283-94. https://doi.org/10.1590/s1413-41522019150122

13. Ministério da Saúde (BR). Portaria de consolidação № 5 , de 28 de setembro de 2017. Consolidação das normas sobre as ações e os serviços de saúde do Sistema Único de Saúde. Diário Oficial União. 29 set 2017.

14. Food and Agriculture Organization of the United Nations FAO. Risk communication applied to food safety handbook. Rome: Food and Agriculture Organization of the United Nations; 2016.

15. Fleetwood J, Rahman S, Holland D, Millson D, Thomson L, Poppy G. As clean as they look? Food hygiene inspection scores, microbiological contamination, and foodborne illness. Food Control. 2019;96:76-86. https://doi.org/10.1016/j.foodcont.2018.08.034

16. Agência Nacional de Vigilância Sanitária - Anvisa. Resolução RDC No 72, de 29 de dezembro de 2009. Dispõe sobre o regulamento técnico que visa à promoção da saúde nos portos de controle sanitário instalados em território nacional, e embarcações que por eles transitem. Diário Oficial União. 30 dez 2009. 
17. Agência Nacional de Vigilância Sanitária - Anvisa. Resolução RDC N 275, de 21 de outubro de 2002. Dispõe sobre o regulamento técnico de procedimentos operacionais padronizados aplicados aos estabelecimentos produtores/industrializadores de alimentos e a lista de verificação das boas práticas de fabricação em estabelecimentos produtores/industrializadores de alimentos. Diário Oficial União. 6 nov 2002.

18. American Public Health Association - APHA. Standard methods for the examination of water and wastewater. 23a ed. Washington: American Public Health Association; 2017.

19. Agência Nacional de Vigilância Sanitária - Anvisa. Resolução RDC $N^{\circ} 216$, de 15 de setembro de 2004. Dispõe sobre regulamento técnico de boas práticas para serviços de alimentação. Diário Oficial União. 16 set 2004.

20. Food and Agriculture Organization of the United Nations FAO. Codex alimentarius commission: procedural manual. 24a ed. Rome: Food and Agriculture Organization; 2015.

21. Mouchtouri V, Malissiova E, Zisis P, Paparizou E, Hadjichristodoulou C. Assessment of hygiene standards and hazard analysis critical control points implementation on passenger ships. Int J Environ Health Res. 2013;23(2):170-9. https://doi.org/10.1080/09603123.2012.708920

22. Capunzo M, Cavallo P, Boccia G, Brunetti L, Buonomo R, Mazza G. Food hygiene on merchant ships: the importance of food handlers' training. Food Control. 2005;16(2):183-8. https://doi.org/10.1016/j.foodcont.2004.01.010
23. West R, Michie S, Rubin J, Amlôt R. Applying principles of behaviour change to reduce SARS-CoV-2 transmission. Nat Hum Behav. 2020;(4):451-9. https://doi.org/10.1038/s41562-020-0887-9

24. Zhang N, Miao R, Huang $\mathrm{H}$, Chan EY. Contact infection of infectious disease onboard a cruise ship. Sci Rep. 2016;6:1-13. https://doi.org/10.1038/srep38790

25. Hadjichristodoulou C, Mouchtouri VA, Guglielmetti P, Lemos $\mathrm{CM}$, Nichols $\mathrm{G}$, Paux T et al. Actions for prevention and control of health threats related to maritime transport in european union. Travel Med Infect Dis. 2013;11(4):238-42. https://doi.org/10.1016/j.tmaid.2013.02.001

26. Caldas JN, Pozzetti VC. Sanitary conditions of the ports of Manaus AM, 2007-2010. Vigil Sanit Debate. 2017;5(3):53-9. https://doi.org/10.22239/2317-269X.0086

27. Castro EH, Silva JL, Formiga JO. Estudo preliminar da potabilidade da água, para consumo humano, da rede de abastecimento do município de Humaitá-AM. In: Anais do $14^{\circ}$ Encontro Nacional de Estudante de Engenharia Ambiental; Brasília, Brasil. Brasília: Universidade de Brasília; 2016.

28. Araújo CF, Hypólito JR, Waichman AV. Assessment the quality of well-water. Rev Inst Adolfo Lutz. 2013;72(1):53-8.

29. Passos EC, Mello ARP, Sousa CV, Alonso ACB, Gonzalez E, Tavares M. Microbiology evaluation of the meal served to passengers in a ship cruiser in the 2009/2010 season: detection of Bacillus cereus in the fish-based meal. Rev Inst Adolfo Lutz. 2010;69(4):571-5.

\section{Agradecimentos}

À Agência Nacional de Vigilância Sanitária (Anvisa) e ao Conselho Nacional de Desenvolvimento Científico e Tecnológico (CNPq).

\section{Contribuiç̧ão dos autores}

Kluczkovski A, kluczkovski-Junior A - Concepção, planejamento (desenho do estudo), interpretação dos dados e redação do trabalho. Pinto S - Aquisição, análises e interpretação dos dados. Pinto M - Concepção, planejamento (desenho do estudo). Caldas J - Concepção, aquisição e interpretação dos dados. interpretação dos dados. Waichman A - Interpretação dos dados. Todos os autores aprovaram a versão final do trabalho.

Conflito de Interesse

Os autores informam não haver qualquer potencial conflito de interesse com pares e instituições, políticos ou financeiros deste estudo.

Licença CC BY-NC atribuição não comercial. Com essa licença é permitido acessar, baixar (download), copiar, imprimir, compartilhar, reutilizar e distribuir os artigos, desde que para uso não comercial e com a citação da fonte, conferindo os devidos créditos de autoria e menção à Visa em Debate. Nesses casos, nenhuma permissão é necessária por parte dos autores ou dos editores. 